\title{
Modelo de indicadores para la evaluación y monitoreo del desarrollo sustentable en la zona costera de Mahahual, Quintana Roo, México' ${ }^{1}$
}

\author{
Model of Indicators for Evaluation and Monitoring of Sustainable \\ Development in the Coastal Area of Mahahual, Quintana Roo, \\ Mexico
}

\footnotetext{
1 Este artículo corresponde al proyecto de investigación "Desarrollo sustentable de la zona costera", desarrollado por el Cuerpo Académico de Geografía y Geomática y Cuerpo Académico de Manejo de Recursos Costeros y Terrestres. Financiado por el Programa de Mejoramiento del Profesorado (PromeP) de la Secretaría de Educación Pública de México.

2 Doctora en Geografía por la UNAM. Profesora-investigadora de la Universidad de Quintana Roo (UQROO), México: loucasti@ uqroo.edu.mx

3 Maestra en Planeación por la UQROO. Profesora-investigadora de la UQROO, México: magv@uqroo.edu.mx

4 Doctora en Geografía por la UNAM. Profesora-investigadora de la UQROO, México: bonnie@uqroo.edu.mx

5 Doctor en Geografía por la UNAM. Profesor-investigador de la UQROO, México: davvelaz@uqroo.edu.mx

6 Doctor en Ciencias Marinas de la Universidad Autónoma de Baja California (UABC). Profesor-investigador de la UABC, México: jlferman@uabc.edu.mx

7 Doctor en Ciencias Marinas de la UABC. Profesor-investigador de la UABC, México: agarcia@uabc.edu.mx

8 Maestra en Geografía por la UNAM. Estudiante del Doctorado en Geografía de la UQROO: malu@uqroo.edu.mx

9 Doctora en Geografía por la Universidad de la Habana. Profesora-investigadora de la UQROO, México: manlagu@uqroo.edu.mx

10 Maestra en Educación por la Universidad Pedagógica Nacional. Profesora-investigadora de la UQROO, México: myeladaqui@ uqroo.edu.mx
} 
Para citar este artículo, utilice el nombre completo así:

Castillo Villanueva, L., González Vera, M. A., Campos Cámara, B. et al. (2014). Modelo de indicadores para la evaluación y monitoreo del desarrollo sustentable en la zona costera de Mahahual, Quintana Roo, México. Perspectiva Geográfica, 19(2), 309-330

\section{Resumen}

La evaluación y el monitoreo del desarrollo sustentable de las zonas costeras son fundamentales para conocer los retos por enfrentar, así como para determinar las estrategias que deban implementarse para su atención. Se propone un marco conceptual y un modelo de indicadores para evaluar el desarrollo de una zona costera. Se generó el índice de desarrollo sustentable para Mahahual obteniendo un valor de 0,40 , que representa un nivel bajo de sustentabilidad, lo que indica que se debe poner especial atención en minimizar los factores de presión que se están presentando específicamente en dos zonas de interacción. Entre los desafíos para la sustentabilidad de Mahahual se encuentra diseñar políticas públicas adecuadas a las condiciones de la región, aplicar correctamente los instrumentos normativos y de planeación del territorio, mejorar la calidad de vida de la comunidad, reducir el impacto de las actividades turísticas y ordenar las actividades y usos en el ambiente marino.

Palabras clave: desarrollo sustentable, esquema presión-Estado-respuesta, indicadores, Mahahual, zona costera.

\section{Abstract}

The evaluation and monitoring of sustainable development of coastal areas is crucial to know the challenges faced and identify strategies to be implemented. A conceptual framework and indicator model was proposed to evaluate the development of coastal areas. Three composite indicators and Sustainable Development Index (SDI) were calculated. The IDS to Mahahual was 0.40 which represents a low sustainability level not in the original text (0.45). From the results, it follows that special attention should be paid to minimize the stress factors that are occurring specifically in two areas of interaction. Among the challenges for the sustainability of Mahahual is designing public policies appropriate to the conditions of the region, correctly applying the regulatory and land use planning instruments to improve the quality of life of the community, reducing the impact of tourism activities and ordering uses and activities in the marine environment.

Keywords: sustainable development, pressure-state-response framework, indicators, Mahahual, coastal zone. 


\section{Introducción}

Una de las grandes preocupaciones a nivel global se centra en cómo hacer frente a los retos del desarrollo sustentable. Esta preocupación se hace más alarmante cuando se incorporan a la ecuación los posibles impactos derivados del cambio climático y las características y dinámicas propias de los espacios geográficos costeros.

Ningún ecosistema sobre el planeta acusa todos los impactos del cambio climático como la zona costera, por lo tanto, es urgente la preparación de la sociedad para los desafíos que enfrentará ahí. El aumento del nivel del mar, la exposición a eventos hidrometeorológicos, la erosión costera, las variaciones en la circulación litoral por la construcción de infraestructura costera, son algunos de los factores que alteran las costas y contribuyen a su vulnerabilidad. Los estudios sobre los impactos del cambio climático en este ámbito han cobrado gran relevancia en una gama amplia de sectores, debido a que las zonas costeras más vulnerables albergan cerca del $10 \%$ de la población mundial. Asimismo, son zonas que, junto con los mares, alojan algunos de los ecosistemas más diversos y productivos del mundo, incluyendo los manglares y los arrecifes de coral. Entre los aspectos que han recibido mayor atención entre la comunidad científica y, por ello, han sido estudiados con mayor profundidad, se encuentran los impactos en la infraestructura costera, en las actividades productivas (por ejemplo, la industria, la pesca, el turismo y las actividades mercantiles) y en los ecosistemas (Yáñez, 2010).

Los impactos del cambio climático son de gran relevancia para México, ya que de las 32 entidades federativas que conforman la República Mexicana, 17 tienen apertura al mar y ocupan una superficie continental total de $1.095 .084 \mathrm{~km} 2$, lo cual representa aproximadamente el $56 \%$ del territorio nacional. Para estos estados se reportó en 2005 una población total de 47.194.599 habitantes, que equivale al $46 \%$ del total nacional. Esta focalización de las poblaciones urbanas en las zonas costeras del país y el volumen de pobladores que en ellas se asientan, plantean serios retos de gestión por las necesidades que estos grupos humanos generan y que deben ser satisfechas, pero también por las alteraciones en el medio ambiente marino y costero que crean (Azuz \& Rivera, 2009). Para 2005, en los municipios costeros del estado de Quintana Roo se informó de una población de 1.097.426 habitantes. Se proyecta que esta cifra llegue a 2.421 .695 en 2030 , lo cual representaría una tasa de crecimiento del $121 \%$ (Seingier, Espejel, Ferman \& Delgado, 2010). El Golfo de México y la costa Caribe de la península 
de Yucatán, están considerados entre los litorales más vulnerables al cambio climático (Yáñez et al., 2010).

Las ciudades merecen atención especial. En primer lugar, porque generan fuertes impactos sobre el ambiente de la zona costera. Sus superficies impermeables resultan en escurrimiento fuertemente contaminado que impacta la calidad de las aguas costeras. Además, la expansión urbana destruye miles de hectáreas de bosques, hábitat costero y humedales en particular. El crecimiento urbano costero desordenado está exponiendo a más y más personas a los peligros costeros, como por ejemplo inundaciones asociadas con huracanes y otras tormentas. El cambio climático solo empeorará esta situación (Islam \& Tanaka, 2004; IAIA, 2001; Levina et al. 2007; citados en Jacob, 2010).

Un elemento fundamental para poder definir e implementar acciones y políticas que permitan enfrentar los retos del desarrollo sustentable de las zonas costeras, es contar con información sobre el estado que guarda el sitio. A su vez, estas acciones y políticas requieren ser evaluadas para determinar su efectividad y eficacia y con base en ello tomar decisiones sobre su continuidad, modificación o cancelación. Por eso, primero se debe conocer cuál es la situación del sitio en un tiempo determinado, para establecer una línea base y a partir de allí llevar a cabo un monitoreo sistemático del proceso de desarrollo sustentable. En este sentido, los indicadores constituyen una herramienta de gestión eficaz que permite dar seguimiento al proceso de desarrollo sustentable, para conocer su evolución en el tiempo y con base en ello tomar decisiones y definir acciones.

Se parte de la hipótesis de que el uso de indicadores para la evaluación y monitoreo del proceso de desarrollo sustentable de las zonas costeras permite conocer cuáles son o podrían ser los retos que deban enfrentarse $\mathrm{y}$ determinar las estrategias por implementar para su atención. Se propone un modelo de evaluación y seguimiento del desarrollo sustentable que considera tres ejes: desarrollo sustentable, modelo PER y espacio geográfico costero.

\section{Marco conceptual para el desarrollo sustentable en zonas costeras}

La constitución histórica del proceso dialéctico naturaleza-ser humano ha derivado en alteraciones a escala mundial, como son la contaminación del aire, agua y suelo y las evidencias de los efectos negativos del cambio climático. De esta relación dialéctica surge el componente transformador "desarrollo", evaluado al principio básicamente mediante indicadores de actividad económica. Este enfoque 
permuta, en la segunda mitad del siglo XX, hacia la relación ambientedesarrollo, como consecuencia de la crisis de un modelo de desarrollo predominante que no contemplaba lo ambiental, surgiendo así el concepto de desarrollo sustentable, con la premisa de satisfacer las necesidades de las presentes generaciones y garantizar - mediante nuevas formas de cooperación social- los cambios requeridos para sostener el progreso humano y la supervivencia del hombre en el planeta (Cantú, 2012). Bolis, Morioka y Sznelwar (2014) proponen que el desarrollo sustentable pueda ser visto como el desarrollo destinado a mejorar el bienestar de la sociedad en su conjunto (incluidas las generaciones futuras), habilitado por una perspectiva ontológica en los procesos de toma de decisiones, teniendo en cuenta las limitaciones de los recursos ambientales. A pesar de las divergencias sobre lo que es el desarrollo sustentable, la discusión ha contribuido, al menos, a la aceptación de que constituye un concepto multidimensional y que es un proceso que implica mejora de calidad de vida y mantenimiento del equilibrio ecológico, más que una condición por alcanzar en un tiempo determinado. Se deben observar las sociedades, los sistemas naturales y su interacción en el transcurrir del tiempo (Bustillo \& Martínez, 2008; Ramírez, Sánchez \& García, 2004).
Por otro lado, se tiene que los indicadores de sostenibilidad y los índices compuestos están tomando mucha importancia y cada vez más como una poderosa herramienta para la formulación de políticas y la comunicación pública de aspectos relacionados con el medio ambiente, la economía y la sociedad. Al conceptualizar los fenómenos y destacar tendencias, los indicadores de sostenibilidad simplifican, cuantifican, analizan y comunican la información compleja (Singh, Murty, Gupta \& Dikshit, 2009).

Uno de los esquemas de indicadores ambientales más aceptados es el PER presión-estado-respuesta, presentado por la OCDE (García, Ferman, Arredondo, Galindo \& Seingier, 2005). El marco conceptual del uso de indicadores que actualmente sugiere la Secretaría de Medio Ambiente y Recursos Naturales (SEMARNAT) para la planificación y manejo de la zona costera, está basado en el modelo de indicadores PER (Azuz et al., 2010). El marco metodológico PER se basa en el concepto de la causalidad: las actividades humanas ejercen PRESIONES sobre el ambiente que cambian la calidad y cantidad de los recursos naturales (ESTADO), finalmente la sociedad RESPONDE a través de políticas ambientales, económicas y sectoriales (OCDE, 1993, citado en García, Ferman, Arredondo, Galin- 
do \& Seingier, 2005). Aplicado al desarrollo sustentable de una región permite establecer las presiones que conducen a una situación determinada de sustentabilidad de la zona, lo cual, a su vez, nos brinda información para la definición de acciones y la toma de decisiones que retroalimenten el modelo para disminuir las presiones $\mathrm{y}$ mejorar el estado social, económico y ambiental. Este esquema, aplicado a lo largo del tiempo, permite evaluar la efectividad de las acciones y las decisiones tomadas.

Respecto a los espacios geográficos costeros, estos son áreas extremadamente frágiles, ya que son el único límite o frontera que separa los tres dominios del planeta: la tierra, el mar y la atmósfera. La definición de zona costera, como elemento clave del sistema costero, es de vital importancia para la comprensión del tema. Conceptualizarla implica considerar varios criterios teniendo en cuenta los puntos de vista ecológico, económico, geográfico, sociológico y jurídico, entre otros. De ahí que las definiciones que de ella se manejan se han elaborado específicamente según las circunstancias para las que vayan a aplicarse (Monzón, 2009). En México estos espacios geográficos presentan también limitaciones jurídicas en cuanto a las atribuciones sobre el territorio. La federación tiene la atribución de elaborar e instrumentar el ordenamiento ecológico marino (OEM), mientras que los municipios tienen la atribución de definir los usos del suelo dentro de su entidad administrativa a través de los programas de ordenamiento ecológico del territorio (POET). Esto genera la división artificial de la realidad de un mismo sistema costero que no abona para el desarrollo sustentable de la región. Los POET a nivel local o regional pueden regular los usos de suelo y actividades productivas de las zonas costeras, pero no las actividades productivas en la Zona Federal Marítimo Terrestre (ZOFEMAT) y el mar (Sánchez, Bocco \& Casado, 2013).

Barragán (1994) señala que el litoral es considerado como el área terrestre contigua a la costa, de amplitud variable según las características o actividades objetos de análisis y la superficie marítima adyacente, también con distintas posibilidades de anchura. Con base en lo anterior se decidió una delimitación con sustento operativo, por lo que en este estudio entenderemos la zona costera de Mahahual como el espacio geográfico conformado por el territorio que ocupa la mancha urbana de Mahahual y las cuatro unidades marinas establecidas en el POET-Costa Maya (Periódico Oficial del Gobierno de Quintana Roo, 2006), que limitan con la localidad.

En 1987, la Comisión Mundial sobre el Medio Ambiente y el Desarrollo hicieron un llamado para la elaboración de nuevas formas de evaluar y 
monitorear el proceso de desarrollo sustentable. Es hasta 1996 cuando un grupo internacional de expertos se reunió en el Centro de Estudios y Conferencias de la Fundación Rockefeller en Bellagio, Italia, y estableció los lineamientos generales para guiar el proceso de evaluación y seguimiento de las acciones de desarrollo sustentable, conocidos como "Los principios de Bellagio". Estos principios incluyen la selección y diseño de indicadores, su interpretación y la comunicación de los resultados: visión y metas orientadoras, perspectiva holística, elementos esenciales, alcance adecuado, enfoque práctico, aperturatransparencia, comunicación eficaz, amplia participación, evaluación continua y capacidad institucional.

La Organización para la Cooperación y el Desarrollo Económico (OCDE) define un indicador como un parámetro, o un valor derivado de parámetros, que indica o proporciona información acerca de, o describe el estado de un fenómeno/ambiente/ área, con significado que va más allá de la que se asocia directamente al valor del parámetro. Sancho y García (2006) mencionan que los indicadores proporcionan un conjunto de señales, regulaciones y datos para la observación de políticas y decisiones gubernamentales. Son medidas de la existencia de dificultades o de la gravedad de las ya conocidas, indicios de situaciones o problemas por venir, medidas del riesgo y de la necesidad potencial de acción y medios para identificar y evaluar los resultados de nuestras acciones.

En el esquema de organización PER, los indicadores de presión describen la influencia que ejercen las diferentes actividades humanas sobre el ambiente y los recursos naturales; los indicadores de estado se refieren a la calidad del ambiente y la cantidad y estado de los recursos naturales y deben estar diseñados para dar información sobre la situación del ambiente y sus cambios a lo largo del tiempo; y los indicadores de respuesta presentan los esfuerzos que realiza la sociedad, las instituciones o gobiernos, orientados a la reducción o mitigación de la degradación del ambiente. En este último tipo de indicadores, por lo general, las acciones de respuesta están dirigidas hacia dos objetivos: 1os agentes de presión y las variables de estado. Si bien la experiencia internacional en la creación y empleo de indicadores e índices es basta, las experiencias específicas sobre zona costera y marina y cambio climático son limitadas (Azuz, Espejel, Rivera, Ferman \& Seingier, 2010).

Hechas las precisiones anteriores, se plantea a continuación el marco conceptual para la evaluación y monitoreo del desarrollo sustentable en zonas costeras, que se compone de dos elementos principales. El primero permite aprehender la realidad que caracteriza las zonas costeras desde la 
perspectiva del proceso de desarrollo sustentable, mediante tres ejes que lo estructuran: el desarrollo sustentable, el modelo PER y el espacio geográfico costero. El desarrollo sustentable analizado a partir de su dimensión social, económica, ambiental e institucional; el esquema PER desde sus componentes de presión, estado y respuesta; y el espacio geográfico costero analizado a partir de sus zonas terrestres y marinas. El segundo, derivado del primero, es precisamente el modelo de indicadores de desarrollo sustentable que facilita la comprensión y seguimiento del proceso de sustentabilidad en una zona costera específica (Figura 1). Los indicadores se definen considerando las combinaciones derivadas de los tres componentes del esquema PER, las cuatro dimensiones del desarrollo sustentable y las dos zonas del espacio geográfico costero.

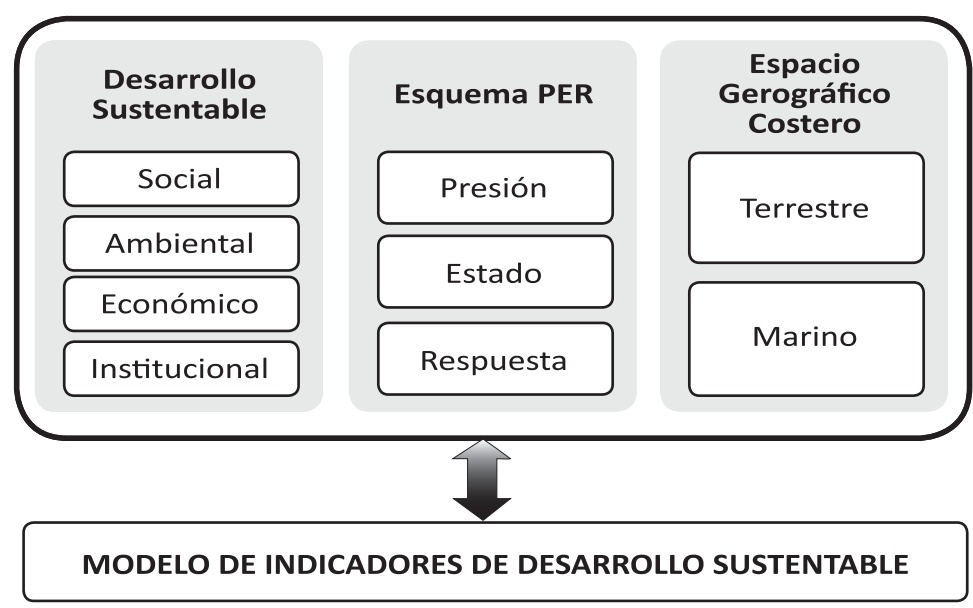

Figura 1. Marco conceptual para la evaluación del desarrollo sustentable en zonas costeras Fuente: elaboración propia.

\section{Zona costera de Mahahual}

La región Costa Maya se localiza en la parte suroriental de la península de Yucatán, que corresponde a la provincia geográfica de la costa baja de Quintana Roo, ocupa una superficie de 98.042 ha, delimita al este con el mar Caribe. La localidad más importante de la región es precisamente Mahahual, asentamiento costero con una población de 920 habitantes (Instituto Nacional de Estadística Geografía e Informática -INEGI-, 2010). La mancha urbana está contenida en el polígono del Centro de Población de Mahahual "UGA CP 17" con una superficie de 1.629,20 ha, de acuerdo con el POET-Costa Maya, decretado en 2000 y actualizado en 2006. Cuenta con una política de aprovechamiento regulada por el Programa Director de 
Desarrollo Urbano cuyos usos compatibles son: asentamiento humano y turismo; usos condicionados: industria, manejo de flora y fauna; y usos incompatibles: acuacultura, agrícola, área natural, corredor natural, forestal, minería, pecuario y pesca. Ambientalmente, Mahahual posee una biodiversidad invaluable, arrecifes de coral, lagunas costeras, manglares, dunas costeras, marismas, flora $\mathrm{y}$ fauna que se encuentran enlistadas en la normatividad ambiental mexicana en algún estado de protección.
El área de estudio (Figura 2) corresponde a la zona costera de Mahahual, conformada por cuatro unidades terrestres: Mahahual Viejo (MV), km 55, Fraccionamiento Mahahual (FM) y Terminal de Cruceros (TC); y cuatro unidades marinas establecidas en el Programa de Ordenamiento Ecológico del Territorio Costa Maya, que limitan con la localidad: UGA Ma05, UGA Ma06, UGA Ma07 y UGA Ma08.

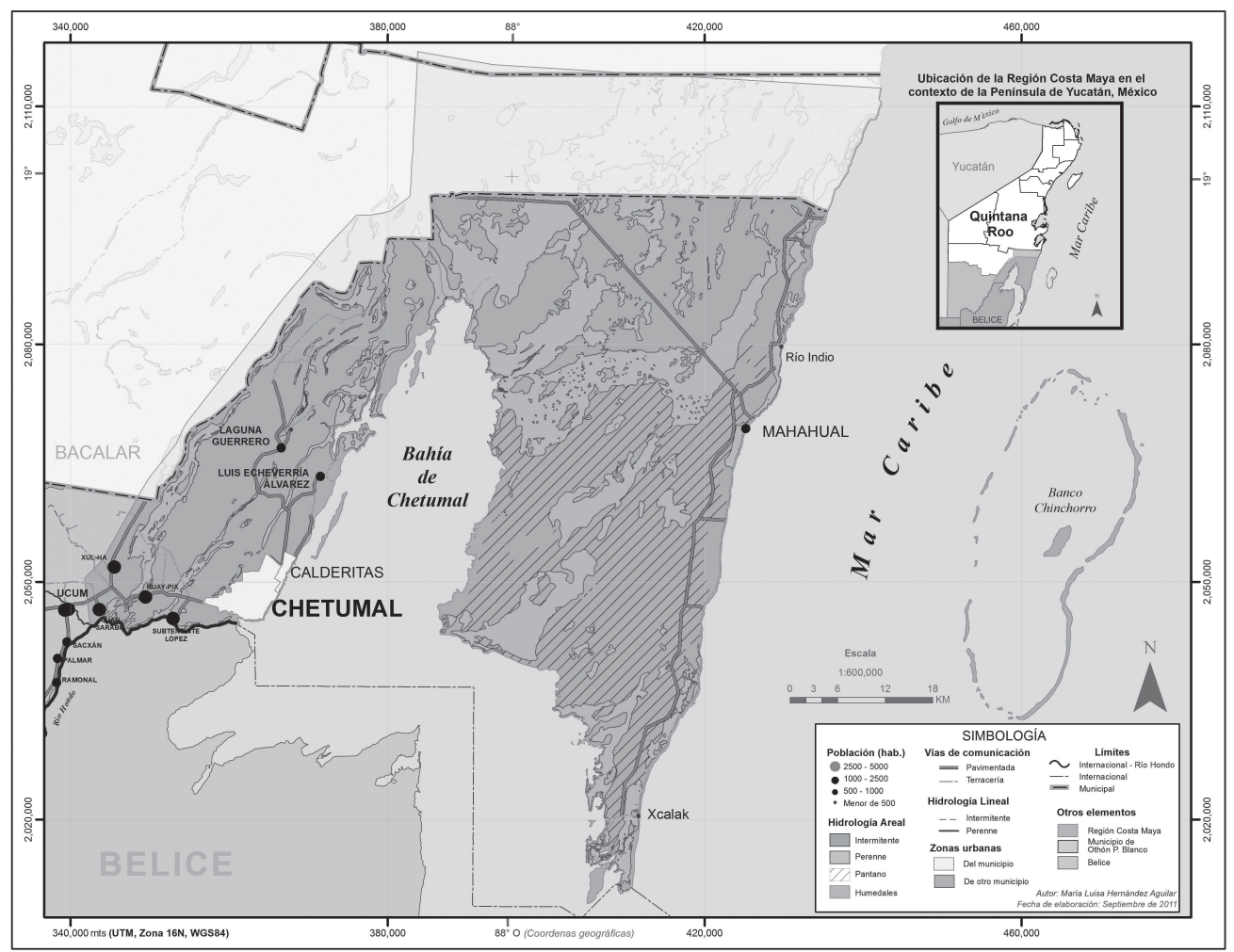

Figura 2. Área de estudio: Mahahual en Costa Maya.

Fuente: elaboración propia. 
3. Modelo de indicadores de desarrollo sustentable en la zona costera de Mahahual

Los indicadores fueron definidos con base en el marco conceptual y clasificados por tipo de indica- dor PER, dimensión del desarrollo sustentable, y tipo de espacio geográfico costero. De lo anterior se obtuvieron 26 indicadores simples: seis de presión, doce de estado y ocho de respuesta (Tabla 1).

Tabla 1. Indicadores simples de desarrollo sustentable

\begin{tabular}{|c|c|c|c|c|}
\hline \multirow{2}{*}{ Tipo PER } & \multirow{2}{*}{$\begin{array}{c}\text { Dimensión Desarrollo } \\
\text { Sustentable }\end{array}$} & \multirow{2}{*}{ Indicador simple } & \multicolumn{2}{|c|}{ Espacio geográfico costero } \\
\hline & & & Terrestre & Marino \\
\hline $\mathrm{P}$ & Económico & Servicios turísticos & $\mathrm{X}$ & $\mathrm{X}$ \\
\hline $\mathrm{P}$ & Económico & Cuartos de hotel & $\mathrm{X}$ & \\
\hline $\mathrm{P}$ & Económico & Actividades turístico-recreativas & $\mathrm{X}$ & $\mathrm{X}$ \\
\hline $\mathrm{P}$ & Económico & Muelles (públicos y privados) & & $\mathrm{X}$ \\
\hline $\mathrm{P}$ & Económico & Pesca comercial y deportiva & & $\mathrm{X}$ \\
\hline $\mathrm{P}$ & Social & Tasa de crecimiento poblacional & $\mathrm{X}$ & $\mathrm{X}$ \\
\hline $\mathrm{E}$ & Social & Servicios urbanos & $\mathrm{X}$ & \\
\hline $\mathrm{E}$ & Social & Equipamientos urbanos & $\mathrm{X}$ & \\
\hline $\mathrm{E}$ & Social & Formas de acceso & $\mathrm{X}$ & $\mathrm{X}$ \\
\hline $\mathrm{E}$ & Ambiental & Cobertura vegetal & $\mathrm{X}$ & \\
\hline $\mathrm{E}$ & Ambiental & Atractivos naturales & $\mathrm{X}$ & $\mathrm{X}$ \\
\hline $\mathrm{E}$ & Ambiental & Especies de corales duros & & $\mathrm{X}$ \\
\hline $\mathrm{E}$ & Ambiental & Especies de corales blandos & & $\mathrm{X}$ \\
\hline $\mathrm{E}$ & Ambiental & Especies de peces & & $\mathrm{X}$ \\
\hline $\mathrm{E}$ & Ambiental & Especies de macroalgas & & $\mathrm{X}$ \\
\hline $\mathrm{E}$ & Ambiental & Pastos marinos & & $\mathrm{X}$ \\
\hline $\mathrm{E}$ & Ambiental & Blanqueamiento de coral & & $\mathrm{X}$ \\
\hline $\mathrm{E}$ & Ambiental & Calidad del agua & & $\mathrm{X}$ \\
\hline $\mathrm{R}$ & Institucional & $\begin{array}{c}\text { Planes y programas de planea- } \\
\text { ción }\end{array}$ & $\mathrm{X}$ & $\mathrm{X}$ \\
\hline $\mathrm{R}$ & Institucional & Restauración de playa & $\mathrm{X}$ & \\
\hline $\mathrm{R}$ & Institucional & $\begin{array}{l}\text { Restauración de vegetación de } \\
\text { duna costera }\end{array}$ & $\mathrm{X}$ & \\
\hline $\mathrm{R}$ & Institucional & Empleo temporal & $\mathrm{X}$ & \\
\hline $\mathrm{R}$ & Institucional & $\begin{array}{l}\text { Mejoramiento de carreteras y } \\
\text { caminos }\end{array}$ & $\mathrm{X}$ & \\
\hline $\mathrm{R}$ & Institucional & Manejo de residuos & $\mathrm{X}$ & \\
\hline $\mathrm{R}$ & Institucional & $\begin{array}{l}\text { Especies de coral con protec- } \\
\text { ción ambiental }\end{array}$ & & $\mathrm{X}$ \\
\hline $\mathrm{R}$ & Institucional & Boyado & & $\mathrm{X}$ \\
\hline
\end{tabular}

Fuente: elaboración propia. 
Con base en los indicadores simples se integraron tres indicadores de desarrollo sustentable por componente
PER y el índice de desarrollo sustentable (Figura 3).

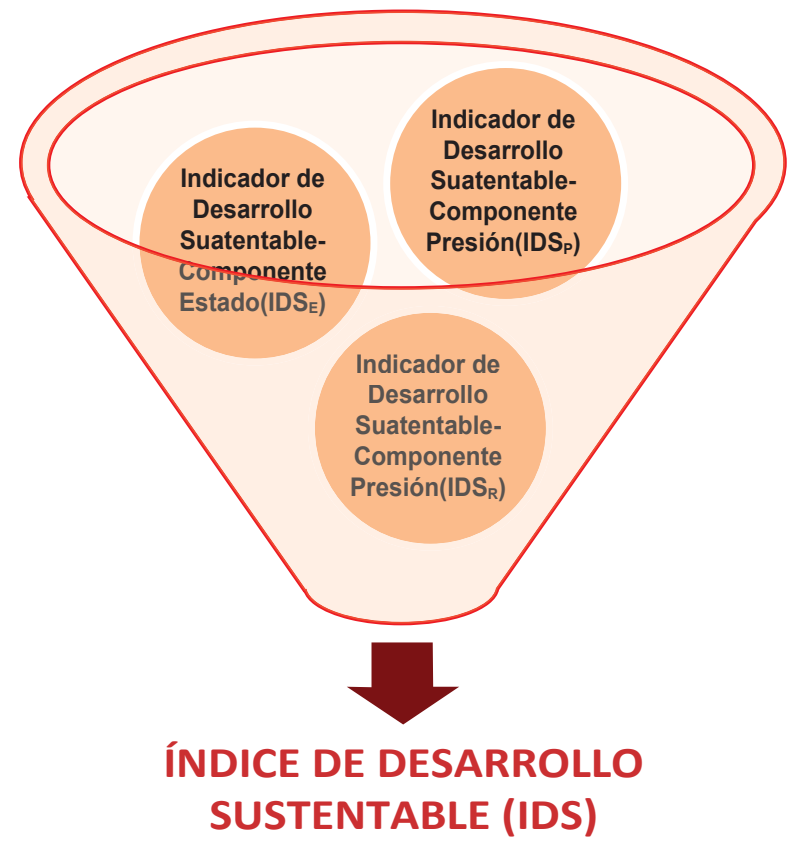

Figura 3. Índice de desarrollo sustentable de la zona costera de Mahahual Fuente: elaboración propia.

\section{Metodología para la construc- ción de indicadores e índice de desarrollo sustentable}

La definición de los indicadores del modelo para el caso de Mahahual se basó en la revisión de indicadores propuestos por diferentes organismos e investigadores (Comisión de Desarrollo Sostenible de las Naciones Unidas -CDS-, 2001; García, 2006; Instituto Brasileiro de Geografia e Estatística -IBGE-, 2002; INEGI \& Instituto Nacional de Eco- logía -INE-, 2000; Quiroga, 2001; Seingier, Espejel \& Ferman, 2009; Sotelo, Tolón \& Lastra, 2011) y en la identificación de las principales problemáticas sentidas por la comunidad. Muchos indicadores no estaban disponibles debido sobre todo a que se presentan a escala nacional o estatal pero no local. Considerando la relevancia y utilidad del indicador, el camino a seguir fue recopilar los datos básicos disponibles para construir un indicador alternativo. Los datos utilizados para el cálculo 
de los indicadores e índice son públicos y fueron obtenidos a partir de tres fuentes: datos oficiales, estudios publicados sobre el área de estudio e imágenes de satélite.

Con base en los indicadores simples se integraron tres indicadores de desarrollo sustentable por componente del esquema PER, los cuales fueron promediados para obtener el índice de desarrollo sustentable. Estos indicadores e índice fueron obtenidos tanto para las unidades terrestres como marinas, espacio geográfico terrestre y marino, y Mahahual.

Para obtener los indicadores de desarrollo sustentable por componente PER, se llevó a cabo lo siguiente: primero los indicadores simples (Tabla 1) fueron clasificados como po- sitivos o negativos de acuerdo con la relación que guardan con la sustentabilidad, en el sentido de si contribuyen o no a su desarrollo; después se determinó el valor mínimo y máximo de cada indicador y se aplicó la fórmula de estandarización correspondiente (Figura 4); por último se obtuvieron los indicadores de desarrollo sustentable por componente PER mediante la suma ponderada de los indicadores estandarizados. Los valores que pueden asumir los indicadores e índices se encuentran en el rango de 0 a 1 , donde, valores cercanos a cero implican una situación no deseada respecto al desarrollo sustentable del área de estudio, al contrario, valores cercanos a uno indican un escenario adecuado de desarrollo sustentable.

$$
\begin{aligned}
& \text { Indicador positivo }=\frac{\text { Indicador simple }- \text { Mínimo }}{\text { Máximo }- \text { Mínimo }} \\
& \text { Indicador negativo }=\frac{\text { Máximo }- \text { Indicador simple }}{\text { Máximo }- \text { Mínimo }}
\end{aligned}
$$

Figura 4. Fórmulas de estandarización Fuente: Castillo, (2009).

Con el fin de poder mapear los resultados obtenidos, estos fueron clasifi- cados en cinco categorías mediante intervalos equidistantes (Tabla 2). 
Tabla 2. Escala de clasificación de indicadores e índice de desarrollo sustentable

\begin{tabular}{|c|c|c|}
\hline Indicador/índice & Posibilidad de desarrollo sustentable & Color \\
\hline $0,805-1,000$ & Muy alta & \\
\hline $0,605-0,805$ & Alta & \\
\hline $0,405-0,605$ & Media & \\
\hline $0,205-0,405$ & Baja & \\
\hline $0,000-0,205$ & Muy baja & \\
\hline
\end{tabular}

Fuente: elaboración propia.

\section{Resultados y discusión}

Se presentan los resultados de los indicadores de desarrollo sustentable por componente presión, estado y respuesta (IDSP, IDSE e IDSR) y el índice de desarrollo sustentable (IDS) desglosados a nivel de cada una de las ocho unidades descritas, espacio terrestre, espacio marino y zona costera de Mahahual (Tabla 3). Valores altos de los indicadores describen mayores posibilidades de avanzar hacia la sustentabilidad, mientras que valores bajos implican menores expectativas de un desarrollo sustentable.

Los IDSP presentan valores que van de 0,33 (baja) en la UGA Ma06, a 0,89 (muy alta) en el km 55. Para el espacio terrestre el valor fue de 0,58 (media) comparado con 0,52 (media) en el espacio marino. A nivel de la zona costera de Mahahual este indicador alcanzó un valor de 0,55 (media). Para el caso de los IDSE los valores más bajos se observaron en el km 55 y Terminal de Cruceros con 0,18 (muy baja) y el valor máximo de 0,65 (alta) en la UGA Ma07. Comparando el espacio terrestre y el marino, los valores fueron de 0,27 (baja) y 0,55 (media) respectivamente. El valor del IDSE correspondiente a Mahahual fue de 0,41 (media). En cuanto a los IDSR el valor más pequeño de 0,00 (muy baja) se registró en la UGA Ma08 y el mayor de 0,68 (alta) en el Viejo Mahahual. Relacionado con el espacio terrestre se obtuvo un valor de 0,43 (media) y con el espacio marino un valor de tan solo 0,08 (muy baja). A nivel de Mahahual el valor fue de 0,25 (baja).

En el caso de los IDS el valor más pequeño fue de 0,28 (baja) y se registró en la Terminal de Cruceros y el más alto de 0,52 (media) en el Viejo Mahahual. Respecto al espacio terrestre, el valor obtenido fue de 0,43 (media) y en el espacio marino fue de 0,38 (baja). El dato para el caso de Mahahual fue de 0,40 (baja), aunque en el límite superior del rango lo que lo ubica en la frontera con el rango medio. 
Los resultados (Tabla 3) de los IDSP, IDSE, IDSR y el IDS fueron mapeados por unidad terrestre y marina de acuerdo con la categori- zación establecida en la Tabla 2, y se obtuvieron las figuras 5, 6, 7 y 8 .

Tabla 3. Resultados de indicadores e índice de desarrollo sustentable de Mahahual

\begin{tabular}{|c|c|c|c|c|c|c|c|c|c|c|c|}
\hline \multirow{2}{*}{ INDICADORES E ÍNDICE } & \multicolumn{4}{|c|}{ Unidades terrestres1 } & \multirow{2}{*}{ ET2 } & \multicolumn{4}{|c|}{$\begin{array}{l}\text { Unidades marinas } \\
\text { (UGA Ma) }\end{array}$} & \multirow{2}{*}{ EM3 } & \multirow{2}{*}{ Mahahua } \\
\hline & 1 & 2 & 3 & 4 & & 05 & 06 & 07 & 08 & & \\
\hline $\begin{array}{l}\text { Indicador de desarrollo } \\
\text { sustentable-componente } \\
\text { presión (IDSP) }\end{array}$ & 0,38 & 0,89 & 0,64 & 0,39 & 0,58 & 0,75 & 0,33 & 0,46 & 0,53 & 0,52 & 0,55 \\
\hline $\begin{array}{l}\text { Indicador de desarrollo } \\
\text { sustentable-componente } \\
\text { estado (IDSE) }\end{array}$ & 0,51 & 0,18 & 0,21 & 0,18 & 0,27 & 0,63 & 0,59 & 0,65 & 0,36 & 0,55 & 0,41 \\
\hline $\begin{array}{l}\text { Indicador de desarrollo } \\
\text { sustentable-componente } \\
\text { respuesta (IDSR) }\end{array}$ & 0,68 & 0,40 & 0,40 & 0,25 & 0,43 & 0,07 & 0,04 & 0,19 & 0,00 & 0,08 & 0,25 \\
\hline $\begin{array}{l}\text { Índice de desarrollo } \\
\text { sustentable (IDS) }\end{array}$ & $\mathbf{0 , 5 2}$ & 0,49 & 0,42 & 0,28 & $\mathbf{0 , 4 3}$ & 0,48 & 0,32 & 0,43 & 0,30 & $\mathbf{0 , 3 8}$ & 0,40 \\
\hline
\end{tabular}

Fuente: elaboración propia.

Los IDSP (Figura 5) muestran que los niveles de presión en el Viejo Mahahual y la Terminal de Cruceros generan bajas posibilidades de sustentabilidad. Los valores son 0,38 y 0,39 . En el espacio marino, la UGA Ma06 y la UGA Ma07 adyacentes a la Terminal de Cruceros y al Viejo Mahahual, son las que menos aportan a la sustentabilidad. Los valores de estas unidades son 0,33 (baja) y 0,46 (media) respectivamente. Entonces las condiciones menos propicias para la sustentabilidad, desde el punto de vista de factores de presión, se presentan en las zonas de contacto terrestre-marino "Terminal de Cruceros-UGA Ma06" y "Viejo Mahahual-UGA Ma07". Estas zonas es donde se lleva a cabo de manera más intensiva la actividad turística, se ofrece la mayor cantidad de servicios turísticos y se ubica el mayor número de infraestructura hotelera.

Considerando los IDSE (Figura 6), se observa que las condiciones en el km 55, Terminal de Cruceros y Fraccionamiento Mahahual, están limitando las posibilidades de sustentabilidad del área, ya que presentan categorías muy bajas y bajas. El km 55 es un asentamiento irregular que se encuentra en proceso de regularización donde aún se carece de servicios y equipamiento adecuado y un porcentaje considerable de cobertura vegetal ha sido eliminado para la 
construcción de viviendas; este último proceso también se ha presentado en el Fraccionamiento Mahahual. Por otro lado, si bien en la Terminal de Cruceros existe disponibilidad de servicios y equipamiento urbano adecua- dos, es en el aspecto ambiental donde se presenta la mayor problemática, ya que la vegetación fue arrasada prácticamente en su totalidad para edificar la Terminal de Cruceros y su infraestructura complementaria.

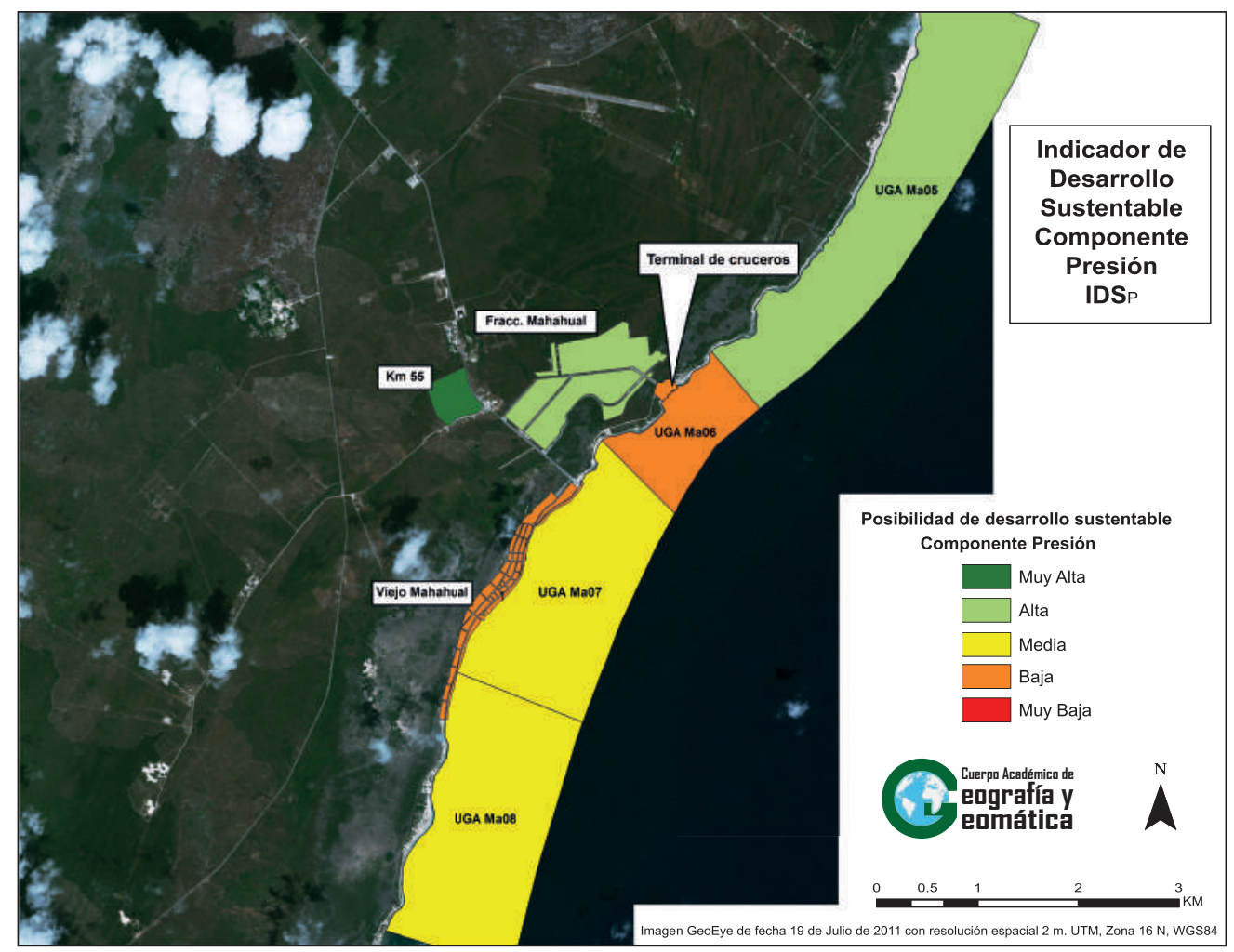

Figura 5. Indicador de desarrollo sustentable-componente presión (IDSP) Mahahual Fuente: elaboración propia.

En general, el IDSE en la zona marina (Figura 6) genera posibilidades medias de desarrollo sustentable de la zona costera. Resalta el hecho de una categoría baja en la UGA Ma05 que se encuentra al sur del área de estudio y que se explica por el blan- queamiento del coral que se encuentra en esa zona.

Los IDSR (Figura 7) permiten evaluar las estrategias y acciones implementadas para la sostenibilidad de la zona costera de Mahahual, sobresale 
la Terminal de Cruceros con un valor de 0,25 (baja) en contraste con

el Viejo Mahahual con un valor de 0,68 (alta).

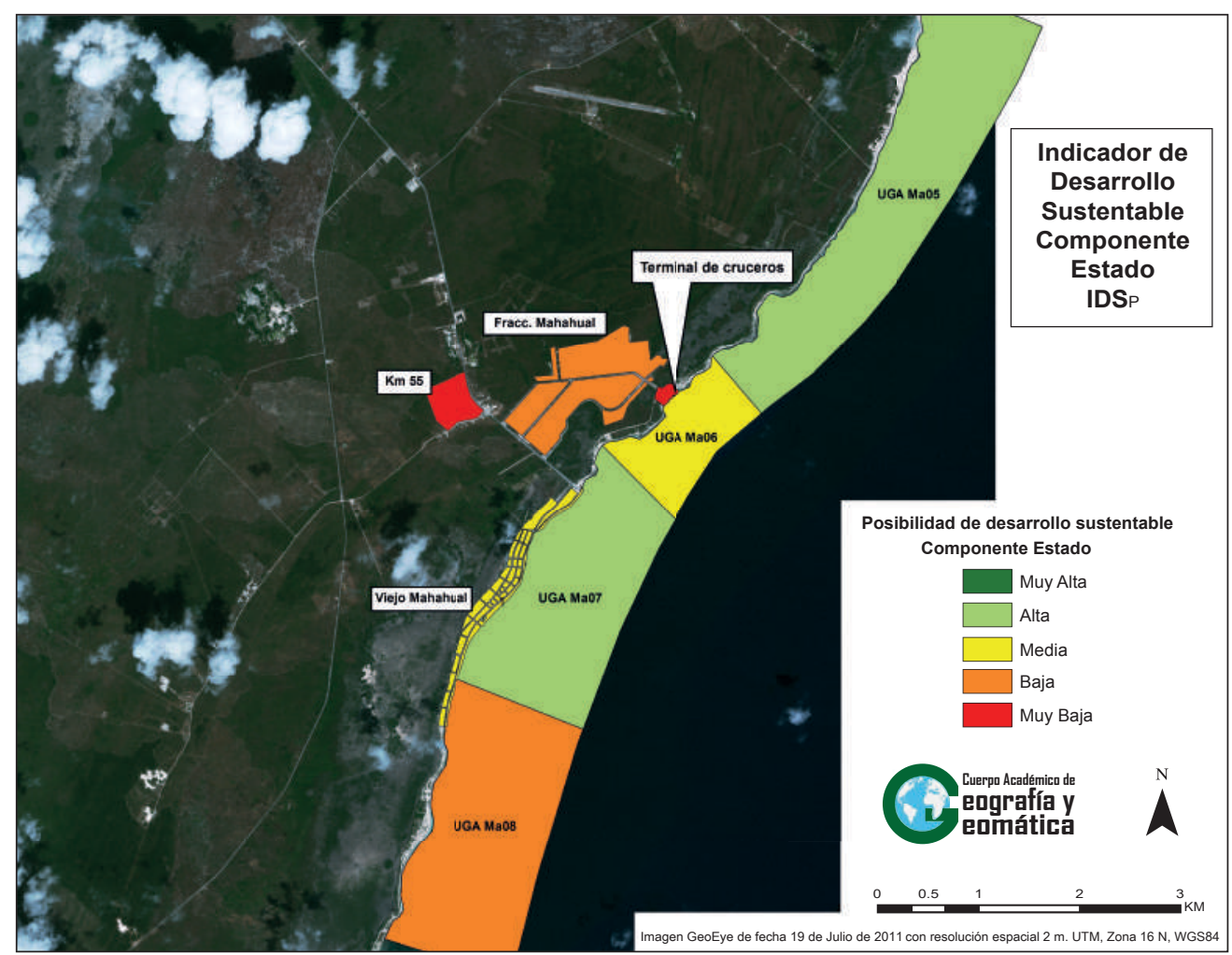

Figura 6. Indicador de desarrollo sustentable-componente estado (IDSE) Mahahual.

Fuente: elaboración propia.

En el espacio marino los IDSR (Figura 7) reflejan la poca atención que se presta desde el punto de vista de la implementación de acciones que busquen reducir las presiones y mejorar sus condiciones (estado), ya que todas las unidades presentan valores inferiores a 0,20 , lo cual significa muy bajas posibilidades de sustentabilidad. La zona de contacto "Terminal de Cruceros-UGA Ma06" vuelve a llamar la atención e indica que no se está prestando la suficiente atención e implementación de medidas de sustentabilidad. El caso de la zona marina se explica por la falta de un ordenamiento marino, ya que si bien existe el POET-Costa Maya, este no tiene incidencia en las unidades marinas por cuestiones normativas. De las unidades terrestres y marinas, la de mayor atención es el Viejo Mahahual con un valor de 0,68 (alta). Esto se puede explicar porque 
el Viejo Mahahual es precisamente el área de principal interés para el sector gubernamental y privado, debido al uso turístico que se hace de la zona, por lo cual se han diseñado acciones para su manejo, conservación y restauración.

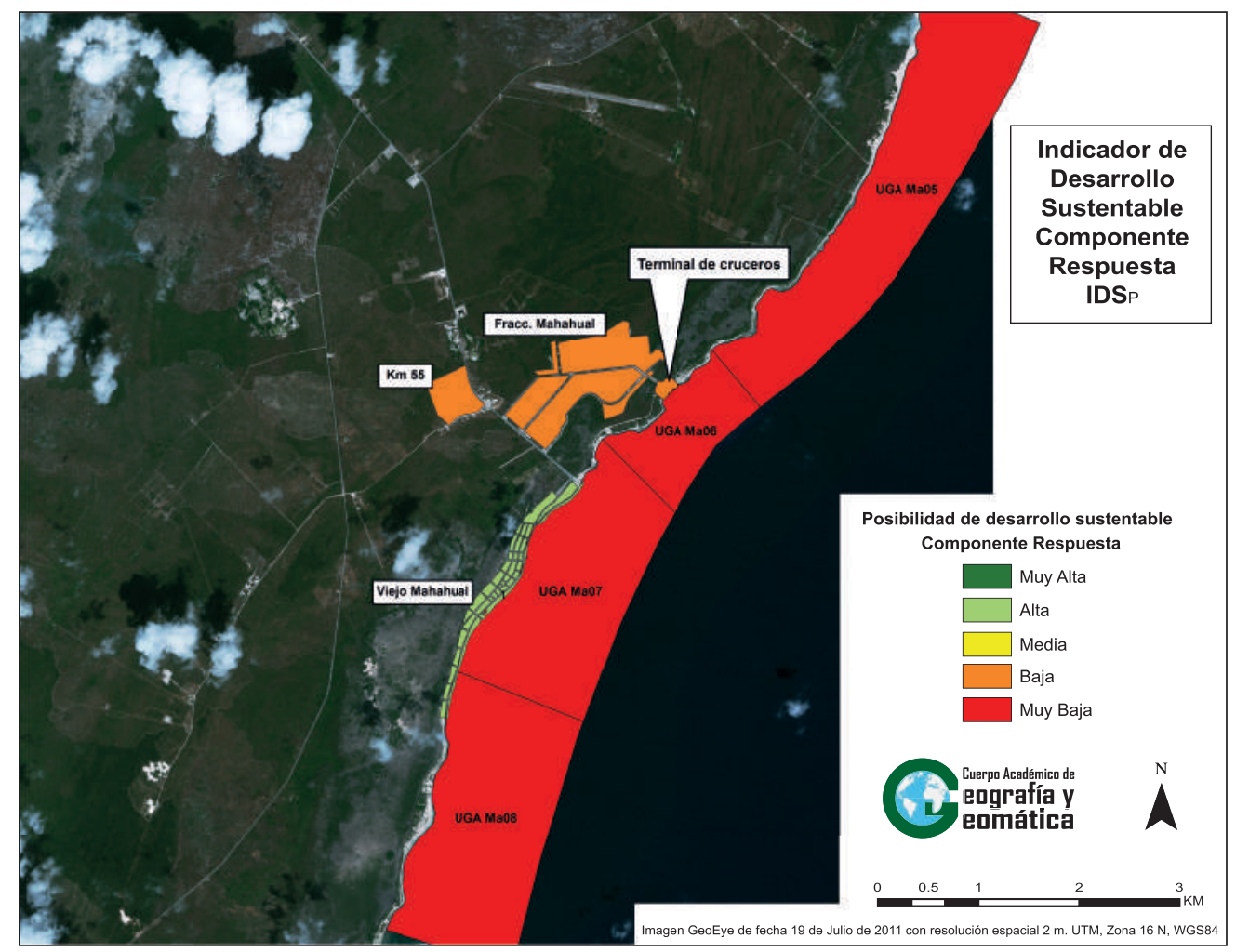

Figura 7. Indicador de desarrollo sustentable-componente respuesta (IDSR) Mahahual Fuente: elaboración propia.

Ahora, al concentrarse en el IDS (Figura 8) se puede observar que el valor más pequeño de 0,28 (baja) corresponde a la Terminal de Cruceros y el valor mayor de 0,52 (media), al Viejo Mahahual. En cuanto al espacio marino, la situación es más homogénea con un valor de 0,30 (baja) en la UGA Ma08 ubicada en la parte sur del área de estudio, un valor de
0,32 en la UGA Ma06 frente a la terminal de cruceros y un valor de 0,48 (media) en la UGA Ma05 ubicada al norte. Estos valores nos indican qué tanto aporta cada una de las unidades al desarrollo sustentable de la zona costera de Mahahual. Al comparar el espacio terrestre y el marino se observa en el primer caso un valor de 0,43 (media) y en el segundo 0,38 
(baja). EI IDS para la zona costera de Mahahual fue de $\mathbf{0 , 4 0}$, lo cual indica un nivel bajo de sustentabilidad, aunque cabe precisar que este valor está muy cercano al límite inferior de la siguiente categoría, por lo cual po- dríamos señalar que de acuerdo con el modelo de indicadores propuesto, el nivel de desarrollo sustentable de Mahahual se puede considerar entre bajo y medio.

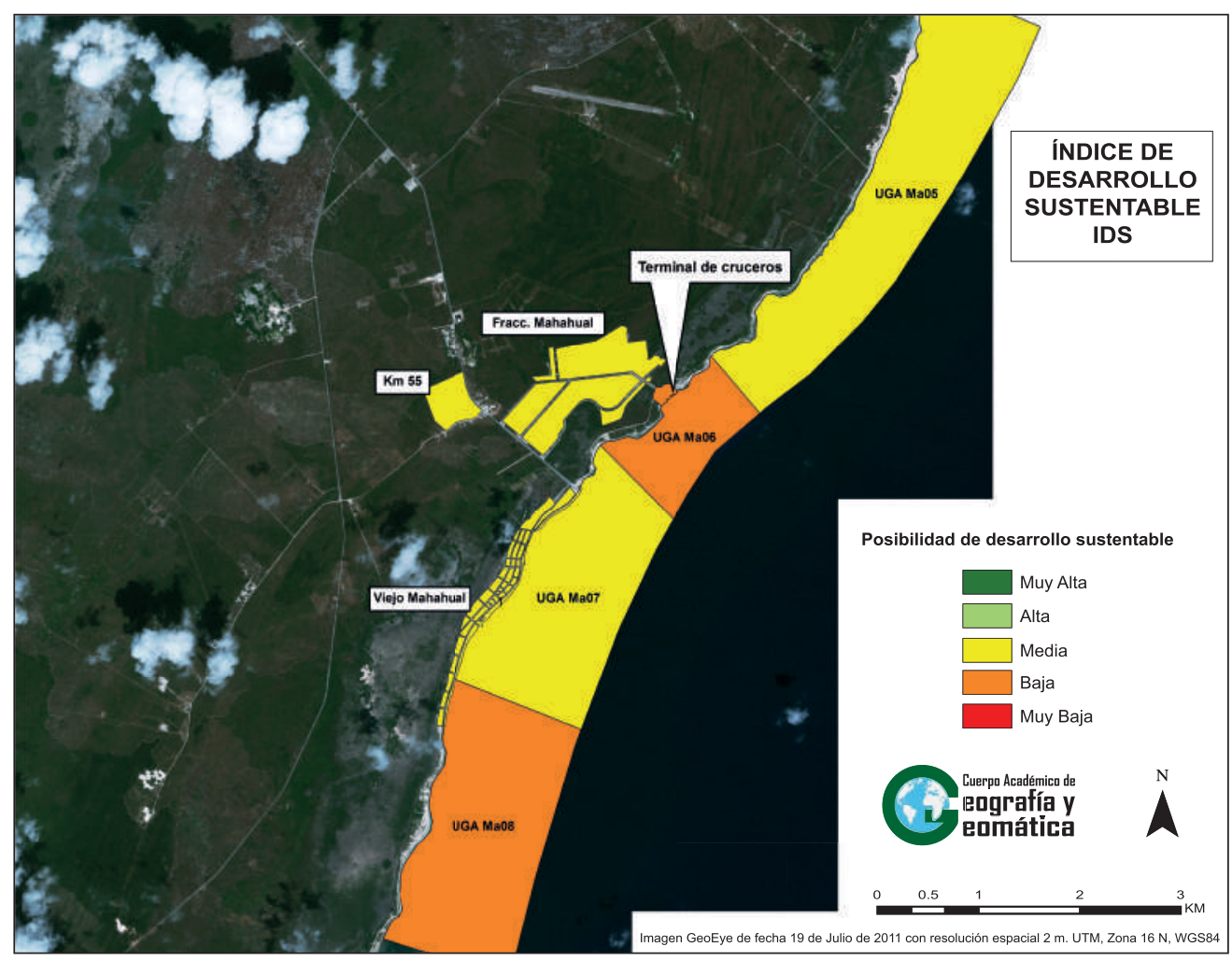

Figura 8. Índice de desarrollo sustentable (IDS) Mahahual Fuente: elaboración propia.

\section{Evaluación del desarrollo} sustentable en la zona costera de Mahahual

Recapitulando lo expuesto en la sección anterior, se puede decir que los resultados se corresponden con la situación presente en Mahahual, lo que muestra la adecuación del modelo de indicadores utilizado para evaluar el potencial de desarrollo en la localidad. El IDSP presenta las menores posibilidades de desarrollo en las unidades terrestres de la Terminal de Cruceros y el viejo Mahahual y la unidad marina UGA Ma06. Esto es 
explicable, ya que son los sitios donde se realiza el mayor número de actividades turísticas, incluyendo la UGA Ma06, donde se ubica el muelle de cruceros, lo que ejerce presión en el área y reduce las posibilidades de un desarrollo sustentable. En cuanto al IDSE los focos rojos se ubican en el km 55 y la Terminal de Cruceros, el primero por carencias sociales y el segundo por cuestiones ambientales. Por otra parte, el IDSR muestra los valores más bajos en todas las unidades marinas, consecuencia de una falta de regulación y de la desvinculación del ordenamiento terrestre respecto al marino. Por último, los resultados para el IDS exponen que las unidades que menos aportan a la sustentabilidad de Mahahual son la Terminal de Cruceros, la UGA Ma06 ubicada frente a la terminal y donde se ubica el muelle de cruceros y la UGA Ma08 debido al blanqueamiento de los arrecifes.

El IDS para Mahahual fue de 0,40, lo cual indica un nivel bajo de sustentabilidad, aunque cabe precisar que este valor está muy cercano al límite inferior de la siguiente categoría, por lo cual podríamos señalar que de acuerdo con el modelo de indicadores propuesto, el nivel de desarrollo sustentable de Mahahual se puede considerar entre bajo y medio.

En Mahahual, como en otras ciudades costeras, las actividades turís- ticas y el crecimiento urbano están generando procesos que no abonan a la sustentabilidad ambiental, social y económica. La comunidad exige una mayor atención gubernamental en los ámbitos de gestión e institucionalidad ambiental, en la definición y aplicación de marcos legales acordes con la dinámica ambiental, social y económica de la región. El modelo de indicadores es un acercamiento a la comprensión del proceso y estado del desarrollo en la zona costera de Mahahual. Permite identificar los retos que deben enfrentarse, lo cual constituye una fuente de información valiosa tanto para los encargados de tomar las decisiones del sector gubernamental como para el sector privado y la sociedad en general. El reto para todos los involucrados en el manejo de la zona costera en Mahahual es enorme. Es necesario diseñar políticas públicas adecuadas a las condiciones de la región, aplicar correctamente los instrumentos normativos y de planeación del territorio, mejorar la calidad de vida de la comunidad y ordenar de forma urgente las actividades en el ambiente marino. Esto último resulta un desafío, ya que la normatividad en la materia ignora que las zonas costeras representan un espacio geográfico complejo donde interactúan ecosistemas terrestres y marinos y por lo cual la planeación del territorio debería ser integral considerando ambos entornos. 
La aplicación del modelo de indicadores a partir del marco conceptual refleja la situación actual de la zona de estudio respecto a un desarrollo sustentable. El marco conceptual podría adoptarse para construir un modelo de indicadores adecuado a otros espacios geográficos no costeros. En el caso de espacios costeros se considera que se podría adaptar el presente modelo de indicadores considerando el contexto y procesos sociales, económicos y ambientales particulares del sitio.

\section{Agradecimientos}

Los autores expresan su agradecimiento al Programa de Mejoramiento del Profesorado por el financiamiento del Proyecto "Desarrollo Sustentable de la Zona Costera”.

\section{Referencias}

Azuz, I., Arredondo, M., Espejel, I., Rivera, E., Seingier, G. \& Ferman, J. (2010). Propuesta de indicadores de la Red Mexicana de Manejo Integrado Costero-Marino. En E. Rivera, I. Azuz, L. Alpuche \& G. Villalobos. (Eds). Cambio climático en México un enfoque costero y marino (pp. 901-939). Campeche: Universidad Autónoma de Campeche, CETYS-Universidad y Gobierno del Estado de Campeche.

Azuz I., Espejel, I., Rivera, E., Ferman, J. \& Seingier, G. (2010). Referentes internacionales sobre indicadores e índices. Historia y estado del arte. En E. Rivera, I. Azuz, L. Alpuche \& G. Villalobos. (Eds). Cambio climático en México un enfoque costero y marino (pp. 845-858). Campeche: Universidad Autónoma de Campeche, CETYSUniversidad y Gobierno del Estado de Campeche.

Azuz, I. \& Rivera, E. (2009). Descripción de la dinámica poblacional en la zona costera mexicana durante el periodo 2000-2005. Papeles de Población, 15(62), 75-107.

Barragán, J. (1994). Ordenación, planificación y gestión del espacio litoral. Barcelona: Oikos-tau.

Bolis, I., Morioka, S. \& Sznelwar, L. (2014). When sustainable development risks losing its meaning. Delimiting the concept with a comprehensive literature review and a conceptual model. Journal of Cleaner Production, 83, 7-20.

Bustillo, L. \& Martínez, J. (2008). Los enfoques del desarrollo sustentable. Interciencia, 33(5), 389-395.

Castillo, L. (2009). Urbanización, problemas ambientales y calidad de vida. México: Plaza y Valdés y Universidad de Quintana Roo.

Cantú, P. (2012). El axioma del desarrollo sustentable. Revista de Ciencias Sociales Universidad de Costa Rica, 3(137), 83-91. 
Comisión de Desarrollo Sostenible de las Naciones Unidas -CDS- (2001). Indicadores de desarrollo sostenible marco y metodologías. Nueva York. Recuperado de http://190.11.224.74:8080/jspui/bitstream/123456789/1190/2/Indicadores de desarrollo sostenible.pdf.

García, A. Ferman, J., Arredondo, M., Galindo, L. \& Seingier, G. (2005). Modelo de planeación ambiental de la zona costera a partir de indicadores ambientales. Sapiens. Revista Universitaria de Invesyigación, 6(2), 9-23.

García, A. (2006). Modelo regional de vulnerabilidad costera. Ensenada: Universidad Autónoma de Baja California.

Instituto Brasileiro de Geografia e Estatística -IBGE- (2002). Indicadores de desenvolvimento sustentável. Río de Janero. Recuperado de ftp://geoftp.ibge.gov.br/documentos/ recursos_naturais/indicadores_desenvolvimento_sustentavel/2012/ids2012.pdf.

Instituto Nacional de Estadística Geografía e Informática -INEGI- (2010). XIII Censo de Población y Vivienda. México: INEGI.

Instituto Nacional de Estadística Geografía e Informática -INEGI- \& Instituto Nacional de Ecología -INE- (2000). Indicadores de desarrollo sustentable en México. México: INEGI \& INE.

Jacob, J. (2010). Urbanización resiliente. Primera respuesta al cambio climático en las costas del Golfo de México. En A. Yañez (Ed.) Impactos del cambio climático sobre la zona costera (pp. 67-90). México: Instituo Nacional de Ecología INE-SEMARNAT e Instituto de Ecología A. C. INECOL.

Monzón, Y. (2009). Derecho y manejo integrado de las zonas costeras. Una aproximación teórica. Ciencia y Sociedad, 34(4), 597-617.

Periódico Oficial del Gobierno de Quintana Roo (2006, 31 de oct.). Decreto mediante el cual se reforma el Programa de Ordenamiento Ecológico Territorial de la Región Costa Maya, Quintana Roo, México, Chetumal.

Quiroga, R. (2001). Indicadores de sostenibilidad ambiental y desarrollo sostenible: estado del arte y perspectivas. Santiago de Chile: CEPAL.

Ramírez, A., Sánchez, J. \& García, A. (2004). El desarrollo sustentable: interpretación y análisis. Revista del Centro de Investigación, 6(21), 55-59.

Sánchez, M., Bocco, G. \& Casado, J. (Eds.) (2013). La política de ordenamiento territorial en México: de la teoría a la práctica. México: UNAM, SEMARNAT e INECC.

Sancho, A. \& García, G. (2006). ¿Qué indica un indicador? Análisis comparativo en los destinos turísticos. Revista de Análisis Turístico, (2), 69-85.

Seingier, G., Espejel, I. \& Ferman, J. (2009). Cobertura vegetal y marginación en la costa mexicana. Investigación ambiental, 1(1), 54-69. 
Seingier, G., Espejel, I., Fermán, J. \& Delgado, O. (2010). Vulnerabilidad de las poblaciones costeras ante la peligrosidad natural, enfoque estatal y municipal. En E. Rivera, I. Azuz, L. Alpuche \& G. Villalobos. (Eds). Cambio climático en México un enfoque costero y marino (pp. 669-688). Campeche: Universidad Autónoma de Campeche, CETYSUniversidad y Gobierno del Estado de Campeche.

Singh, R, Murty, H., Gupta, S. \& Dikshit, A. (2009). An overview of sustainability assessment methodologies. Ecological Indicators, 9(2), 189-212.

Sotelo, J., Tolón, A. \& Lastra, X. (2011). Indicadores por y para el desarrollo sostenible, un estudio de caso. Estudios Geográficos, 72(271), 611-654.

Yáñez, A. (Ed.) (2010). Impactos del cambio climático sobre la zona costera. México: Instituto Nacional de Ecología INE-SEMARNAT e Instituto de Ecología A.C. INECOL.

Yáñez, A., Way, J., Jacob, J., Ibáñez, C., Martínez, A., Miranda, A., Tejeda,A., Welsh, C. \& Carranza, A. (2010). Panel INECOL 2008 -Conclusiones- La zona costera y sus impactos ecológicos, económico y sociales. En A. Yáñez (Ed). Impactos del cambio climático sobre la zona costera (pp. 173-179). México: Instituo Nacional de Ecología INE-SEMARNAT e Instituto de Ecología A. C. INECOL.

Recepción: 18 de noviembre de 2013

Evaluación: 16 de marzo de 2014

Aprobación: 14 de abril de 2014 